\title{
Wavelet Based Image Compression, Pattern Recognition And Data Hiding
}

\author{
${ }^{1}$ P. Aparna, ${ }^{2}$ K. Praveena, ${ }^{3}$ Bhavani. V \\ Associate professor, Assistant professor, Assistant professor \\ Department of ECE, M.V.J.College of Engineering, Bangalore, Karnataka.
}

\begin{abstract}
Images require substantial storage and transmission resources, thus image compression is advantageous to overcome these difficulties. This paper presents Application of DWT for image processing. The main objectives of this paper are image compression, reconstruction, pattern recognition and image data hiding.

First, in compression of two-dimensional image data is divided an image into blocks of size $N \times M$ and then computed the DWT of each block at the finest spatial resolution. The blocks of transformed coefficients were classified into type HH, HL, LH and LL. Again, block LL can be used to classify four more blocks in second level. Each class consisted of group of blocks of size (N/2) x (M/2) of similar type. Then these blocks are quantized using SPIHT (Set Partitioning in Hierarchical Trees) Algorithm to get a compressed image (i.e. bit stream). Reconstruction of image follows exactly reverse process i.e. inverse SPIHT and inverse DWT. The reconstructed image will be equivalent to original image.

New algorithms are developed for pattern recognition and image data hiding, Secondly DWT based feature for pattern recognition. This algorithm is based on binary comparison using X-OR operation and Hamming distance algorithms. Since here binary comparison is done, the DWT is used to enable better recognition accuracy.

Finally, this paper presents image data hiding. In this, compressed image (bit stream image or called signature image) is embedded into a dummy image (or called host image). This is done using bit replacement technique. The quality of embedded image cannot be identified by human visual system.
\end{abstract}

\section{Introduction}

Uncompressed images require considerable storage capacity and transmission bandwidth. The only solution is to compress image before its storage and transmission, and decompress it at the receiver for play back. The scope of the paper is to analyze the wavelet decomposition for source encoding in image compression system, Also deals with the recognition of numerical data images used in defense applications, automatic detection of vehicles, and data hiding.

An Image (color, BMP Picture) is divided into blocks of size N x M and DWT of each block is computed. DWT outputs four types of block, each of size (N/2) x (M / 2). The four types of blocks are labeled, HH, HL, LH and LL, from four classes, each consists of similar blocks. The coefficient matrix of each blocks of size $(\mathrm{N} / 2) \mathrm{x}(\mathrm{M} / 2)$ are quantized. Set Partitioning In Hierarchical Tree (SPIHT) algorithm used as a quantizer. The SPIHT gives bit stream as output. Reconstruction of an image is reverse process i.e. first quantized image has to be converted back to blocks and then reverse DWT will give original image. For pattern recognition Hamming distance algorithm is used to make decision. It compares the given number is matching with the already compressed numbers. Finally, for image hiding bit replacement scheme is used. Any image in image embedding scheme is concerned with the following criteria, namely, how to embed a large number of bits into the image, and not perceptually distort the image at the same time. After the signature data is embedded in the host image, the resulting image is called the embedded image, which is ideally supposed to be perceptually indistinguishable from the host image.

\section{Discrete wavelet transform}

The figure below shows how an image can be decomposed using sub-band. Take an N X M image and filter each row and then down sample to obtain two $\mathrm{N} \mathrm{X} \mathrm{M/2} \mathrm{images.} \mathrm{Then} \mathrm{each} \mathrm{column} \mathrm{is} \mathrm{filtered} \mathrm{and}$ subsample the filter output to obtain four N/2 X M/2 images. Of the four sub images, the one obtained by lowpass filtering the rows and columns is referred to as the LL image; the one obtained by low-pass filtering the row and high-pass filtering the columns is referred to as the LH image; the one obtained by high-pass filtering the rows and low-pass filtering the columns is called the HL image; and the subimage obtained by high-pass filtering the rows and columns is referred to as HH image. Each of the subimages obtained in this fashion can then be filtered and subsampled to obtain our more subimages. This process can be continued until the desired subband structure is obtained. 


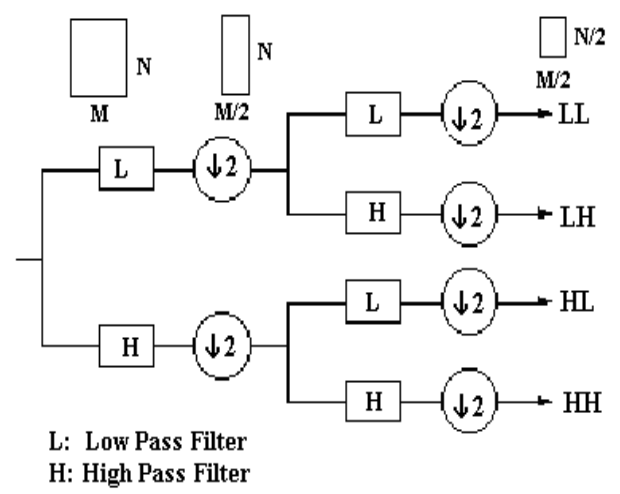

Figure 1 Separable 4-subband of an image

SPHIT(Set Partitioning In Hierarchical Trees)

III. The Proposed Algorithm
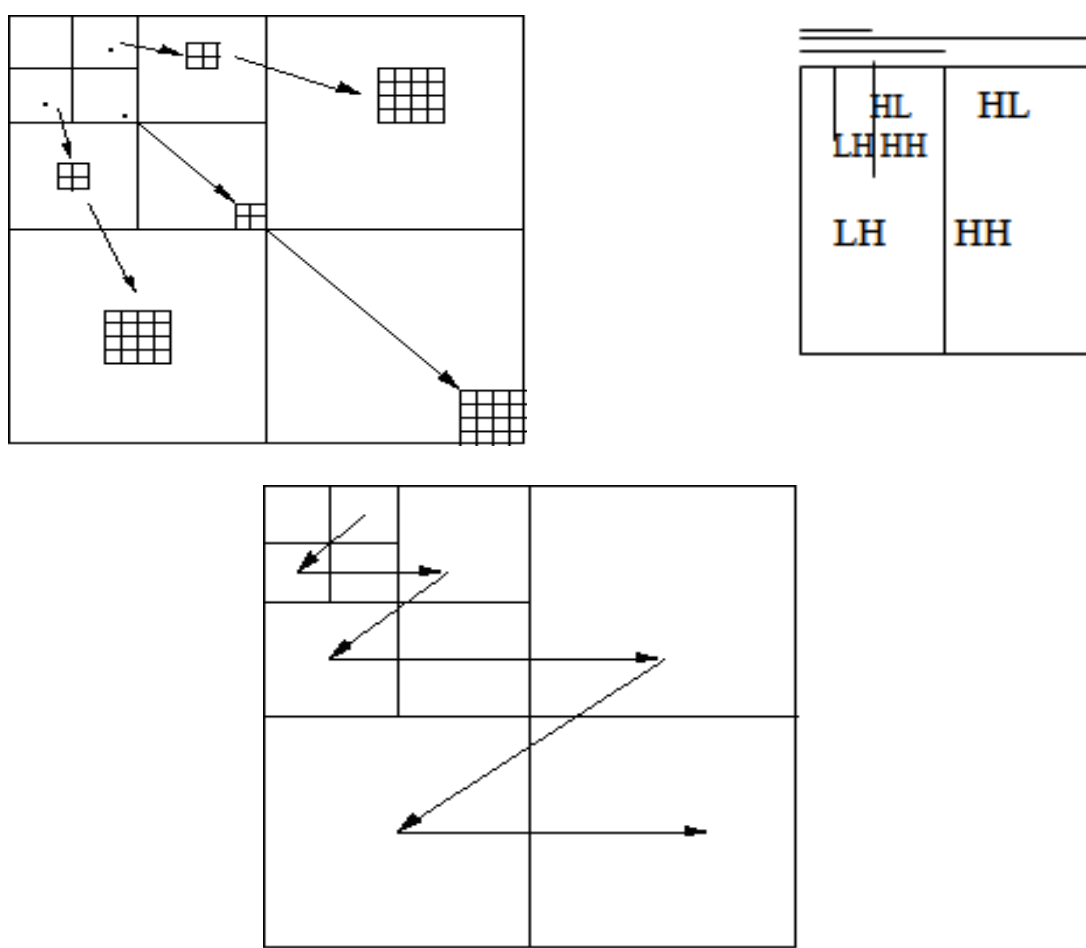

Figure2 (a) Structure of SPIHT, and (b) Scanning order of subbands for encoding

The set partitioning sorting algorithm uses the following four sets of coordinates:

1. $O(i, j)$ : This is the set of coordinates of the offsprings of the wavelet coefficient at location $(i, j)$. As each node can either have four offsprings or none, the size of $(i, j)$ is either zero or four.

2. $D(i, j)$ : This is the set of all descendents of the coefficient at location $(i, j)$. Descendents include the offsprings, the offsprings of the offsprings, and so on.

3. $H(i, j):$ This is the set of coordinates of the roots of all the spatial orientation trees.

4. $L(i, j)$ : This is the set of coordinates of all the descendents of the coefficient at location

$(i, j)$ except for the immediate offsprings of the coefficient at location $(i, j)$. In other words, $L(i, j)=D(i, j)-O(i$, j).

A set $D(i, j)$ or $L(i, j)$ is said to be significant if any coefficient in the set has a magnitude greater than the threshold. Finally, thresholds used for checking significance are powers of two, so in essence the SPIHT algorithm sends the binary representation of the integer value of the wavelet coefficients. The bits are numbered with the least significant bit being the $0^{\text {th }}$ bit, the next bit being the first significant bit, and the $k^{\text {th }}$ bit being referred to as the $k-1$ most significant bit.This algorithm makes use of three lists: the list of insignificant pixels (LIP), the list of significant pixels (LSP), and the list of insignificant sets (LIS). The LSP and LIS lists will 
contain the coordinates of coefficients, while the LIS will contain the coordinates of the root of sets of type D or L. We start by determining the initial value of the threshold. We do this by calculating -

$\mathrm{n}=\left[\log _{2} \mathrm{C}_{\max }\right]$

Where $\mathrm{C}$ max is the maximum magnitude of the coefficients to be encoded. The LIP list is initialized by with the set $\mathrm{H}$. Those elements of $\mathrm{H}$ that have descendents are also placed in LIS as type D entries. The LSP list is initially empty. In each pass, we will first process the members of LIP, then the members of LIS. This is essentially the significance map encoding step. We then process the elements of LSP in the refinement step. We begin by examining each coordinate contained in LIP. If the coefficient at that coordinate is significant (that is, it is greater than $2^{\mathrm{n}}$ ), we transmit a 1 followed by a bit representing the sign of the coefficient (we will assume 1 for positive, 0 for negative). We then move that coefficient to LSP list. If the coefficient at that coordinate is not significant, we transmit a 0. After examining each coordinate in LIP, we begin examining the sets in LIS. If te set at coordinate $(i, j)$ is not significant, we transmit a 0 . If the set is significant we transmit a 1 . What we do after that depends on whether the set is of type $\mathrm{D}$ or L. If the set is of type $\mathrm{D}$, we check each of the offsprings of the coefficient at that coordinate. In other words, we check the four coefficients whose coordinates are $\mathrm{O}(\mathrm{i}, \mathrm{j})$. For each coordinate that is significant, we transmit a 1, the sign of the coefficient, and then move the coefficient to the LSP. For the rest we transmit a 0 and add their coordinates to the LIP. Now that we have removed the coordinates of $\mathrm{O}(\mathrm{i}, \mathrm{j})$ from the set, what is left is simply the set $\mathrm{L}(\mathrm{i}, \mathrm{j})$. If this set is not empty, we move it to the end of LIS and mark it to be of type L. Note that this new entry into the LIS has to be examined during this pass. If the set is empty we remove the coordinate $(\mathrm{i}, \mathrm{j})$ from the list.If the set is of type $\mathrm{L}$, we add each coordinate in $\mathrm{O}(\mathrm{i}, \mathrm{j})$ to the end of LIS as the root of a set of type D. Again, note that these new entries in the LIS have to be examined during this pass. We then remove $(i, j)$ from the LIS. Once we have processed each of the sets in the LIS (including the newly formed once), we proceed to the refinement step. In the refinement step we examine each coefficient that was in the LSP prior to the current pass and output the nth most significant bit of $\left|\mathrm{C}_{\mathrm{ij}}\right|$. This completes one pass.

\section{a. Results for compression \\ i. Results for Lena}

IV. Results

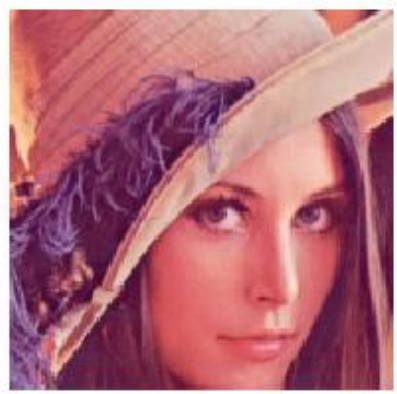

(a)

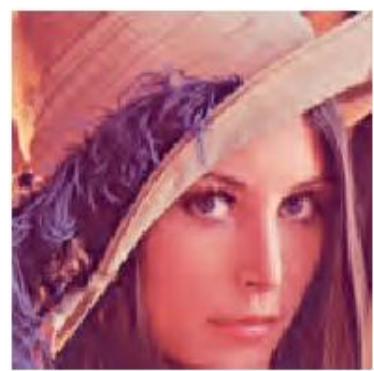

(b)

Figure 3 (a) Original Lena. (b) Reconstruction of compressed with discrete wavelet.

\section{ii. Results for Pattern recognition}

The objective of this paper is to recognize the numeric image and can be extended to recognize vehicles, building finding, boat recognition images. The picture below is an example of the results I have gotten. If the following numeric BMP image is having small distortion even then the project will help in recognizing image. 


\section{8}

Figure 4 A numeric BMP image

\section{c. Results for Image Hiding}

Before embedding the compressed image into dummy image.

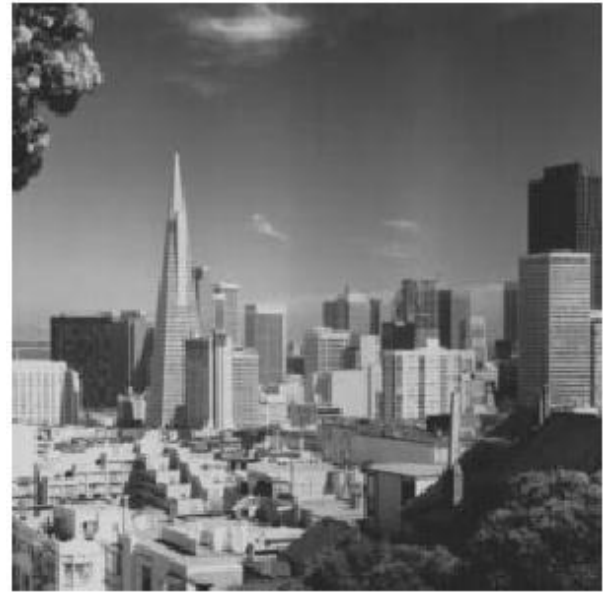

(a)

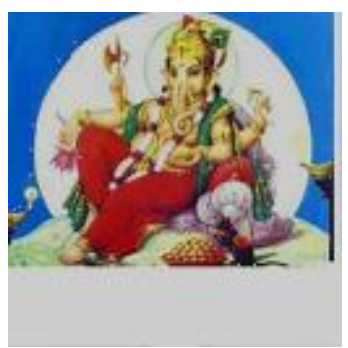

(b)

Figure 5 Dummy (host image) image for hiding. (b) Compressed image (signature image).

After embedding compressed image into dummy image we get the dummy image as shown below.

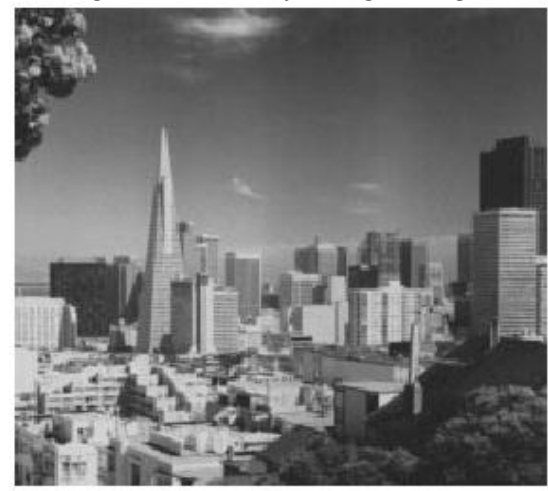

Figure 6 Embedded image.

\section{Conclusion}

While the DCT-based image coders perform very well at moderate bit rates, at higher compression ratios, image quality degrades because of the artifacts resulting from the block-based DCT scheme. Waveletbased coding on the other hand provides substantial improvement in picture quality at low bit rates because of overlapping basis functions and better energy compaction property of wavelet transforms. Because of the inherent multi-resolution nature, wavelet-based coders facilitate progressive transmission of images thereby allowing variable bit rates. The upcoming JPEG-2000 standard will incorporate many of these research works 
and will address many important aspects in image coding. This process of compression reduces the redundancy in the image and important thing is the quality of the reconstructed image will be almost equivalent to the original image. So there will be an efficient performance of compression and decompression of the images. The other feature involve pattern recognition which is aimed to get the best result by making use of binary comparison technique i.e. the failure cases will be very less. As said earlier hiding process is the very significant feature which encapsulates the compressed image into another dummy image where the human visual perception cannot identify the difference.

\section{References}

[1] Eric J. Stollnitz, Tony D. Derose, and David H. Salesin, “Wavelets for computer graphics”, 1996 Morgan Kaufmann Publishers, Inc.

[2] C. Sidney Burrus, Ramesh A. Gopinath, and Haitao Guo, "Introduction to Wavelets and Wavelet Transforms", 1998 prenticeHall,Inc.

[3] M.Rao and A. S. Bopardikar, Wavelet Transforms: “Introduction to Theory and Applications”, 1998 Addison Wesley Longman, Ptd.

[4] Said and W. A. Pearlman, "A new, fast, and efficient image codec based on Set Partitioning in Hierarchical Trees." IEEE trans. Circuits and sysyems for video technology, vol. 6,june 1996.

[5] Froment, J. and Mallat, S. "Wavelets: A Tutorial in Theory and Applications", vol. 2, Academic Press, NY, 1992.

[6]. Vetterli, M. and Kovacevic, J. "Wavelets and Subband Coding", Englewood Cliffs, NJ, Prentice Hall, 1995, http://cm.belllabs.com/who/jelena/Book/home.html.

[7]. Robi Polikar, "The Engineer's Ulimate Guide to Wavelet Analysis"; Thw Wavelet Tutorial http://engineering.rowan.edu/ polikar/WAVELETS/WTtutorial.html

[8]. Amara Graps, "An introduction to Wavelets", IEEE Computational Sciences and Engineering, Volume 2, Summer1995.http://www.amara.com/IEEEwave/IW history.html

[9]. The Purdue JPEG tutorial htt://dynamo.ecn.purdue.edu/ ace/jpeg-tut/jpegtutl.html

[10]. Rarael C. Gonzalez, and Richard E. Woods, "Digital Image Processing",1999 Addison Weseley Longman, Pte Ltd.

[11]. G. M. Davis,"The Wavelet Image Compression 1996. http://www.cs.dartmouth.edu/ gdavis/wavelet/wavelet.html

[12]. R.A. DeVore, B. Jawerth, and B.J. Lucier,'Image Compression Through Wavelet Transform Coding", IEEE Trans. Image Processing, Vol.1, 1992.

[13]. Strang, G. and Nguyen, T."Wavelets and Rilter Banks", Wellesiey-Cambridge Press, Wellesley, MA, 1996, http://wwwmath.mit.edu/ gs/books/wfb.html.

[14]. MOFFAT, A., NEAL, R.M., WITTEN, I.H.,"Arithmetic coding revisited", ACM Transaction on Information Systems, July 1998.

[15]. ANTONINI M. et al.,’Image Coding Using Wavelet Transform”. IEEE Transactions on Image Processing,1992 